\title{
Effects of repeated milnacipran and fluvoxamine treatment on mechanical allodynia in a mouse paclitaxel-induced neuropathic pain model
}

\author{
Soh Katsuyama, Kazuma Sato, Tomomi Yagi, Yukinaga Kishikawa, and Hitoshi Nakamura \\ Department of Clinical Pharmaceutics, Tohoku Pharmaceutical University, 4-4-1 Komatsushima, Aoba-ku, Sendai 981-8558, Japan \\ (Received 10 December 2012; and accepted 25 January 2013)
}

\begin{abstract}
Paclitaxel is widely used in cancer chemotherapy for the treatment of solid tumors, but it frequently causes peripheral neuropathy. Milnacipran, a serotonin/noradrenaline reuptake inhibitor and fluvoxamine, a selective serotonin reuptake inhibitor, have shown efficacy against several chronic pain syndromes. In this study, we investigated the attenuation of paclitaxel-induced mechanical allodynia in mice by milnacipran and fluvoxamine. Paclitaxel was administered once per day $(2 \mathrm{mg} / \mathrm{kg}$, intraperitoneally (i.p.)) for 5 days to mice. Mechanical allodynia was evaluated by measuring the withdrawal response to stimulation with a von Frey filament. In paclitaxel-treated mice, mechanical allodynia was observed on days 3-15 of paclitaxel administration. A single administration of milnacipran $(20 \mathrm{mg} / \mathrm{kg}$, i.p.) or fluvoxamine $(40 \mathrm{mg} / \mathrm{kg}$, i.p.) had no effect on paclitaxel-induced mechanical allodynia. However, repeated administration of milnacipran (10, $20 \mathrm{mg} / \mathrm{kg}$, once per day, i.p.) for 5 days significantly reduced paclitaxel-induced mechanical allodynia. In contrast, repeated fluvoxamine administration $(40 \mathrm{mg} / \mathrm{kg}$, once per day, i.p.) for 5 days resulted in a weak attenuation of paclitaxel-induced mechanical allodynia. These results suggest that chronic paclitaxel administration induces mechanical allodynia, and that repeated milnacipran administration may be an effective therapeutic approach for the treatment of neuropathic pain caused by paclitaxel treatment for cancer.
\end{abstract}

Peripheral neurotoxicity induced by antineoplastic drugs (taxanes, vinca-alkaloids and platin-based compounds) is a clinically significant complication that can be dose-limiting and can substantially diminish quality of life. Paclitaxel is commonly used for the treatment of solid tumors and ovarian and breast cancers. Paclitaxel induces antimitotic actions by impeding the cell cycle in the late phases of mitosis, stabilizing microtubule formation, and ultimately inducing apoptosis (26). However, paclitaxelinduced peripheral neuropathy is a side-effect of chemotherapeutic treatment which often manifests

Address correspondence to: Dr. Hitoshi Nakamura Department of Clinical Pharmaceutics, Tohoku Pharmaceutical University, 4-4-1 Komatsushima, Aoba-ku, Sendai 981-8558, Japan

Tel: +81-22-727-0211, Fax: +81-22-727-0149

E-mail: nakamura@tohoku-pharm.ac.jp as bilateral pain in the extremities in a stocking and glove-type distribution (6), sometimes greatly impairing the patients' quality of life and their ability to perform normal activities of daily living. To date, therapeutic drugs have been evaluated using various animal models of paclitaxel-induced allodynia. Ethosuximide (7), gabapentin (14), thalidomide and minocycline (3), neurotropin (11) and acetyl-L-carnitine (8) have been examined against the paclitaxelinduced neuropathic pain model, although studies regarding their efficacy have not been conclusive.

Supplementary analgesics (antidepressants and anticonvulsants) have suppressive effects on opioid-resistant neuropathic pain $(16,24)$, and antidepressants are widely used for the treatment of neuropathic pain. Antidepressants, especially tricyclic antidepressants (TCAs), are regarded as first-line drugs for the treatment of neuropathic pain (16). Recently, more selective monoamine reuptake inhibitors, such as 
serotonin (5-HT)/noradrenaline (NA) reuptake inhibitors (SNRI) and selective serotonin reuptake inhibitors (SSRI), have become available and are used clinically as neuropathic pain modalities (5). Newer antidepressants such as those in the SNRI and SSRI classes have been introduced, and their antidepressant function is better understood than that of TCAs.

The antinociceptive effects of SSRIs have been reported in a number of animal experiments $(12,13$, $25)$. One typical SSRI, fluvoxamine, attenuated licking behavior in the late phase of the formalin test $(22,30)$ and also in the hot-plate test (27), but it failed to attenuate mechanical allodynia in a neuropathic pain model (chronic constriction injury model) (9). Moreover, fluvoxamine showed an antiallodynic effect in a streptozotocin-induced diabetic neuropathy model (9). Similar to the research on SSRIs, the analgesic effects of SNRIs have also been examined using a variety of animal models of pain (18). One typical SNRI, milnacipran, has been shown to be efficacious for the prevention and/or reversal of pain in several preclinical models of acute and chronic pain in rodents. Yokogawa et al. demonstrated that milnacipran significantly attenuated late phase paw licking behavior in the formalin test (30) and also reversed mechanical allodynia in the chronic constriction injury model or spinal nerve ligation model of neuropathic pain $(9,28)$. In addition, milnacipran had an antiallodynic effect on the streptozotocin-induced diabetic neuropathy model (9) and potentiated the antihyperalgesic effect of tramadol (opioid analgesic) (21). However, the antiallodynic effects of milnacipran and fluvoxamine on paclitaxel-induced mechanical allodynia remain unknown.

The principal aim of this work was to test whether single or chronic administration of milnacipran and fluvoxamine would produce antinociception in the context of the paclitaxel-induced neuropathic pain model in mice.

\section{MATERIALS AND METHODS}

Animals. Male ddY-strain mice (Japan SLC, Hamamatsu, Japan) weighing an average of $23-25 \mathrm{~g}$ at the time of testing were used in these experiments. Mice were housed individually in a colony maintained in a controlled environment (12 h light/dark cycle, room temperature $23^{\circ} \mathrm{C}, 50-60 \%$ relative humidity). The animals had unlimited access to food pellets and water. All behavioral experiments took place during the light period between 10:00 and $16: 00$ in a quiet room. The animals belonging to the various treatment groups ( $n=10$ each group) were tested in randomized order. All experiments followed the Guidelines on Ethical Standards for Investigation of Experimental Pain in Animals (32). Additionally, the study was approved by the Committees of Animal Care and Use of Tohoku Pharmaceutical University.

Experimental protocol. Paclitaxel (Bristol-Myers Squibb Company, Tokyo, Japan) was administered intraperitoneally (i.p.) to mice at doses of $0.5-2 \mathrm{mg} /$ $\mathrm{kg}$, once per day for 5 consecutive days. Paclitaxel dosing was determined based on previous reports that used mice $(4,19)$. Milnacipran hydrochloride or fluvoxamine maleate (Sigma Chemical Co., St. Louis, MO) was administered i.p. to mice. All drugs were dissolved in physiological saline, in an injection volume of $0.1 \mathrm{~mL} / 10 \mathrm{~g}$ body weight. In the vehicle control group, physiological saline was administered by the same schedule. Mechanical allodynia of the hind paw was assessed using von Frey filaments with $0.4 \mathrm{~g}$ bending force. Briefly, mice were placed individually in a plastic cage with a wire mesh bottom. After mice had adapted to the testing environment for $60 \mathrm{~min}$, a von Frey filament with $0.4 \mathrm{~g}$ bending force was pressed perpendicularly against the mid-plantar surface of the hind paw from below the mesh floor and held for 3-5 s with the filament slightly buckled. Lifting of the paw was recorded as a positive response. Stimulation of the same intensity ( $0.4 \mathrm{~g}$ filament) was applied to the point of bending ten times to the plantar surface of the hind paw of each mouse at intervals of $5 \mathrm{~s}$. Behavioral testing was performed between $10: 00$ and $16: 00 \mathrm{~h}$. Testing was performed for two days before the start of the experiment to accustom the mice to the testing procedures.

Data analysis. Data are presented as means \pm S.E.M. values. The statistical significance of the differences between groups was determined using the Student's $t$-test or one-way analysis of variance (ANOVA) followed by Dunnett's test, or two-way ANOVA followed by Bonferroni's test. The level of statistical significance was set at 5\% $(P<0.05)$ in all experiments. Statistics were performed using GraphPad Prism software (version 3; Graph Pad Software, Inc, San Diego, CA).

\section{RESULTS}

Time course and dose-response of paclitaxel-induced mechanical allodynia

Paclitaxel or vehicle (saline) was administered to 
mice once per day for 5 days (days $0-4$ ). We evaluated withdrawal responses to mechanical stimulation applied to each hind paw using a von Frey filament $(0.4 \mathrm{~g})$ from day 0 to day 30 in ddY mice. Mechanical allodynia was not observed when paclitaxel was administered intraperitoneally at a dose of $0.5 \mathrm{mg} /$ $\mathrm{kg}$. When the dose was increased to 1 or $2 \mathrm{mg} / \mathrm{kg}$, mechanical allodynia became marked (Fig. 1A). Thus, we subsequently used $2 \mathrm{mg} / \mathrm{kg}$ as the working dose of paclitaxel to induce mechanical allodynia. With repeated daily dosing of 1 or $2 \mathrm{mg} / \mathrm{kg}$ of paclitaxel, mechanical allodynia peaked 10 days after the start of administration, then decreased gradually, almost subsiding by day 20. In paclitaxel-treated mice, mechanical allodynia was significant on days 3-15, compared with the saline control group (Fig. 1B). In contrast to the repeated dosing regimen, mechanical allodynia was not observed after a single administration of paclitaxel $(2 \mathrm{mg} / \mathrm{kg}$; data not shown).

The effect of paclitaxel on the overall health of the mice appeared to be minimal. No mouse presented with weight loss or died within 30 days after administration.

Effects of milnacipran or fluvoxamine treatment on paclitaxel-induced mechanical allodynia

A single administration of milnacipran $(20 \mathrm{mg} / \mathrm{kg}$, i.p.) or fluvoxamine $(40 \mathrm{mg} / \mathrm{kg}$, i.p.) had no effect on paclitaxel-induced mechanical allodynia on day 10 (Fig. 2A and 2B). However, repeated milnacipran administration $(5-20 \mathrm{mg} / \mathrm{kg}$, once per day, i.p.) for 5 days (days 0-4), in combination with paclitaxel, significantly attenuated paclitaxel-induced mechanical allodynia on day 5 in a dose-dependent manner (Fig. 3A). Moreover, repeated milnacipran administration (5-20 mg/kg, once per day, i.p.) on days 5-9 (after cessation of paclitaxel treatment) significantly attenuated paclitaxel-induced mechanical allodynia on day 10 in a dose-dependent manner (Fig. 3B). In contrast, repeated fluvoxamine administration $(40 \mathrm{mg}$ / $\mathrm{kg}$, once per day, i.p.) for 5 days (days $0-4$ ) or days 5-9), resulted in a weak attenuation of paclitaxel-induced mechanical allodynia on day 5 or 10 (Fig. 4A and $4 \mathrm{~B})$.

\section{DISCUSSION}

Neuropathic pain is defined as pain initiated or caused by a primary lesion or dysfunction in the nervous system (15). Neuropathic pain is often reported as having a lancinating or continuous burning character and is often associated with the appear- ance of abnormal sensory signs, such as allodynia (pain as a result of a stimulus which does not normally induce pain) or hyperalgesia (an increased response to a stimulus which is normally painful) (29).
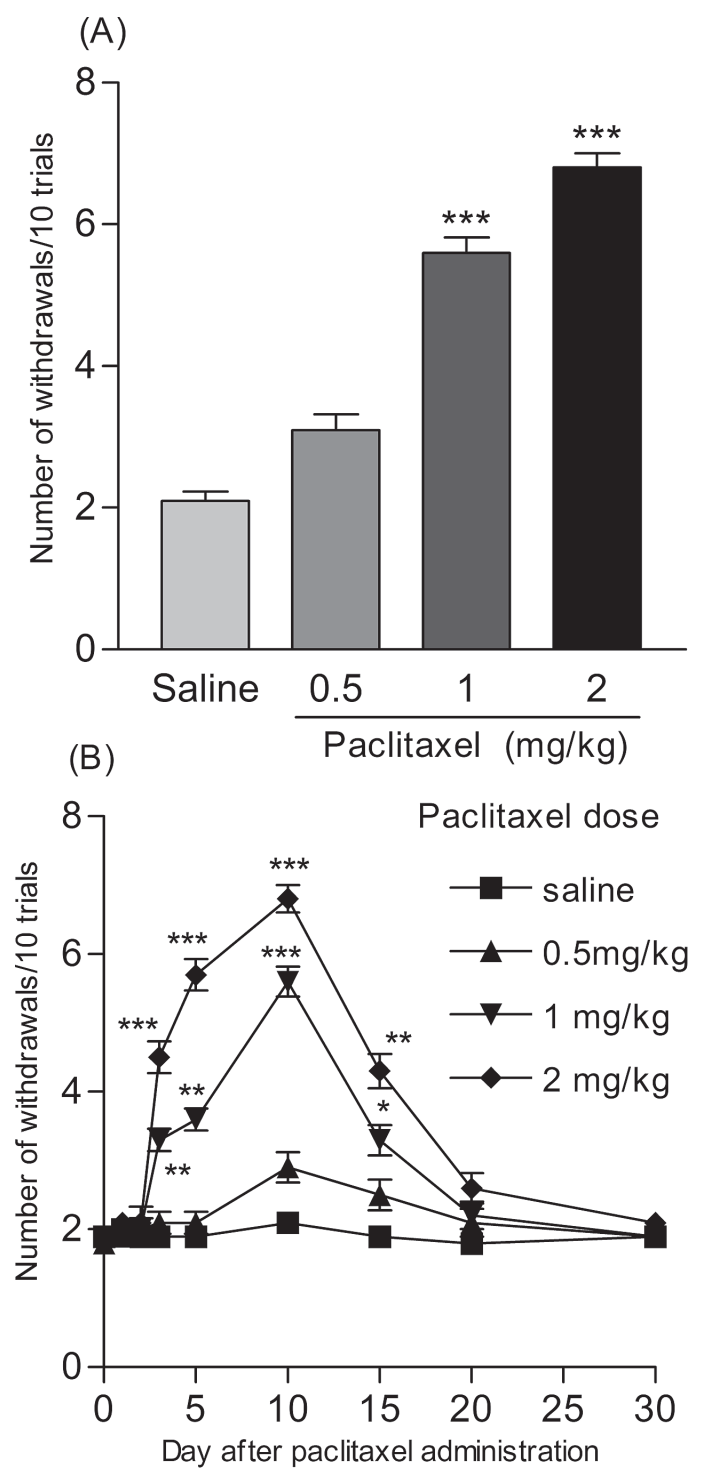

Fig. 1 Paclitaxel (0.5-2 mg/kg, i.p.) or saline was administered once per day for 5 days (days $0-4$ ) to mice. (A) On day 10 , the number of withdrawals to mechanical stimulation using a von Frey filament $(0.4 \mathrm{~g})$ was measured. (B) Time course of paclitaxel-induced mechanical allodynia. The number of withdrawals to mechanical stimulation using a von Frey filament $(0.4 \mathrm{~g})$ was measured on days 1, 2, 3, 5, $10,15,20,30$, prior to daily paclitaxel administration. Data were calculated as the mean \pm S.E.M. values of 10 mice in each group. The statistical significance of the differences between the groups was assessed with one-way ANOVA or two-way ANOVA followed by Dunnett's test (A) or Bonferroni's test (B), respectively. ${ }^{*} P<0.05,{ }^{* *} P<0.01,{ }^{* \star *} P<0.001$ when compared with the saline control group. 

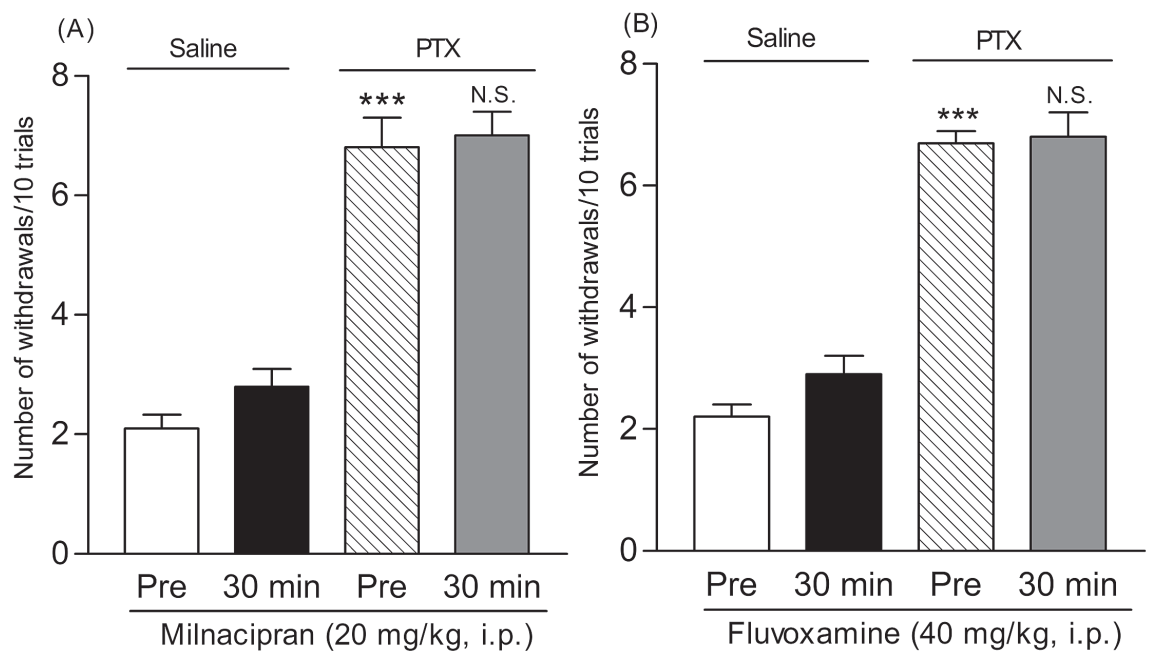

Fig. 2 Paclitaxel (PTX; $2 \mathrm{mg} / \mathrm{kg}$, i.p.) or saline was administered once per day for 5 days (days $0-4$ ) to mice. On day 10 , the number of withdrawals to mechanical stimulation using a von Frey filament $(0.4 \mathrm{~g})$ was measured at 0 min (Pre) and $30 \mathrm{~min}$ after a single administration of milnacipran (20 mg/kg, i.p.) (A) or fluvoxamine (40 mg/kg, i.p.) (B). Data were calculated as the mean \pm S.E.M. values of 10 mice in each group. The statistical significance of the differences between the groups was assessed with Student's $t$-test. ${ }^{* * *} P<0.001$ when compared with the saline control group, N.S.; not significant when compared with the paclitaxel pre-milnacipran group (PTX-Pre).
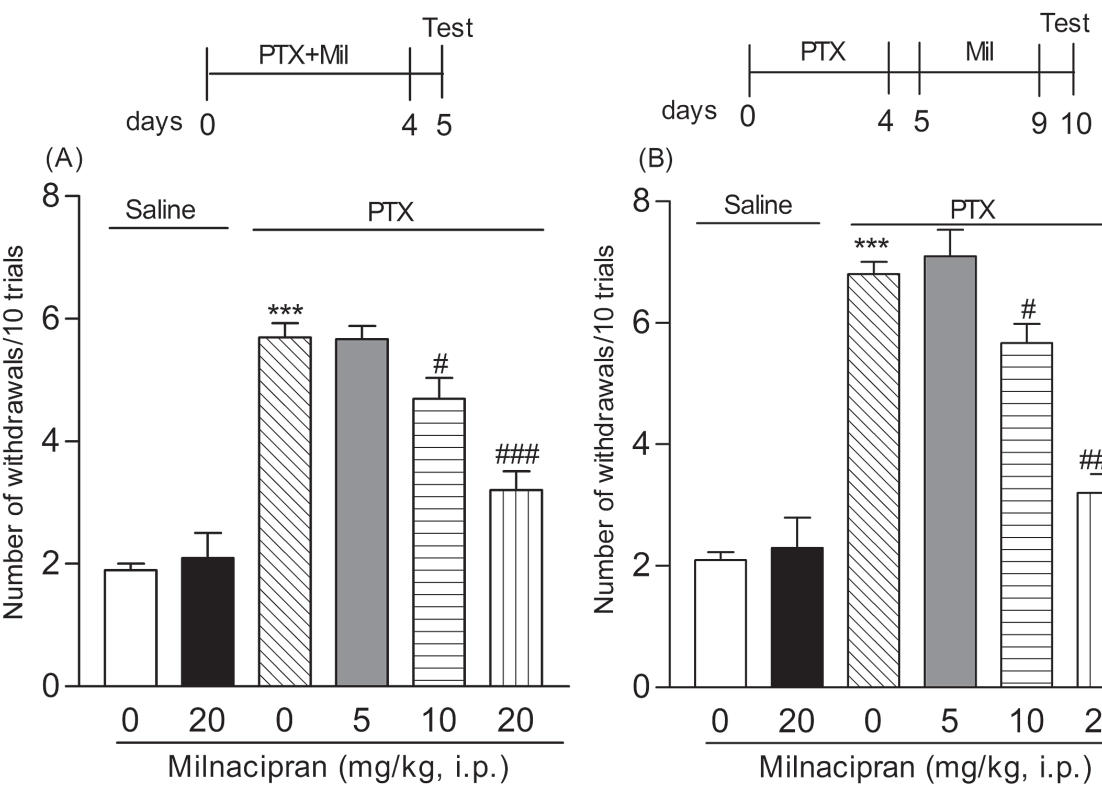

(B)

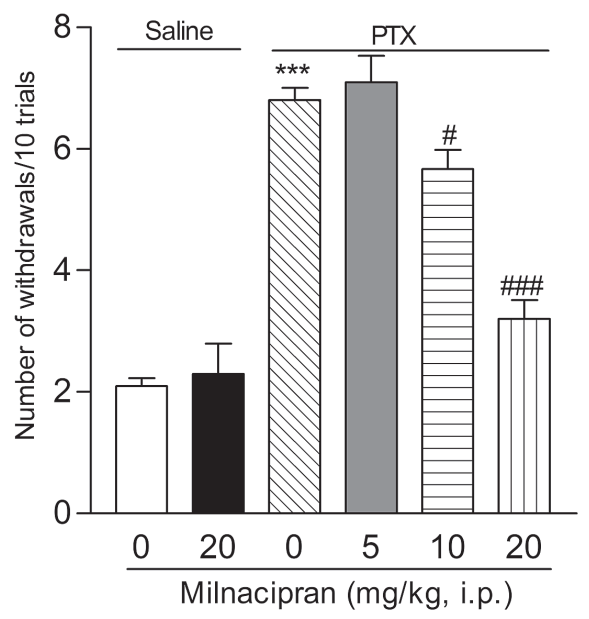

Fig. 3 (Top) Schedule of drug administration. (Bottom) Paclitaxel (PTX; 2 mg/kg, i.p.) and milnacipran (Mil; 5, 10, 20 mg/kg, i.p.) were co-administered once per day for 5 days (days $0-4$ ) to mice (A), or milnacipran (Mil; 5, 10, $20 \mathrm{mg} / \mathrm{kg}$, i.p.) was administered to mice once per day for 5 days (days $5-9)$ after cessation of PTX (2 mg/kg, i.p.) treatment (B). On day 5 (A) and day $10(B)$, the number of withdrawals to mechanical stimulation using a von Frey filament $(0.4 \mathrm{~g})$ was measured. Data were calculated as the mean \pm S.E.M. values of 10 mice in each group. The statistical significance of the differences between the groups was assessed with one-way ANOVA followed by Dunnett's test. ${ }^{* \star *} P<0.001$ when compared with the saline control group, ${ }^{\#} P<0.05,{ }^{\# \# \#} P<0.001$ when compared with the PTX-group.

In this study, we demonstrated that repeated but not acute administration of paclitaxel ( 1 or $2 \mathrm{mg} / \mathrm{kg}$, i.p.) produced dose-dependent mechanical allodynia in male ddY-strain mice. The mechanical allodynia was observed even after cessation of paclitaxel administration in this experimental model (Fig. 1A and 

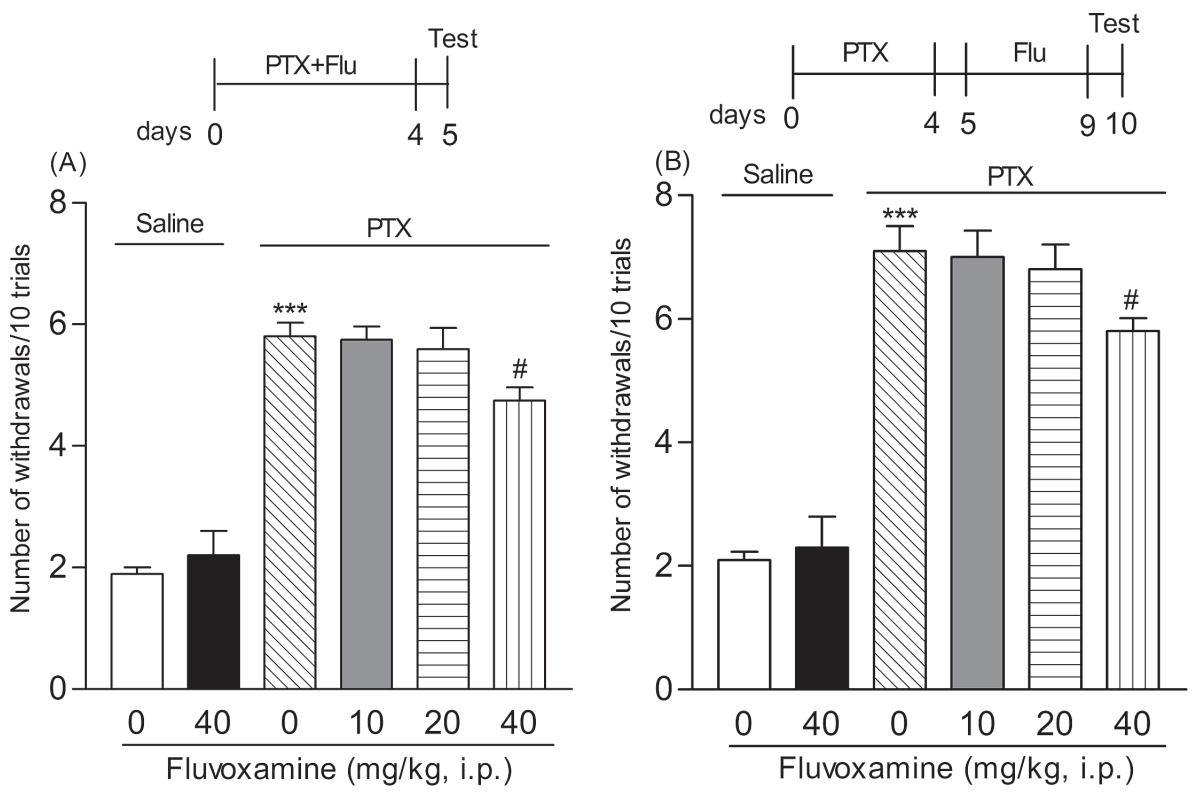

Fig. 4 (Top) Schedule of drug administration. (Bottom) Paclitaxel (PTX; $2 \mathrm{mg} / \mathrm{kg}$, i.p.) and fluvoxamine (Flu; 10, 20, $40 \mathrm{mg} /$ kg, i.p.) were co-administered once per day for 5 days (days $0-4)$ to mice (A) or fluvoxamine (Flu; 10, 20, $40 \mathrm{mg} / \mathrm{kg}$, i.p.) was administered to mice once per day for 5 days (days 5-9) after cessation of PTX (2 mg/kg, i.p.) treatment (B). On day 5 (A) and day 10 (B), the number of withdrawals to mechanical stimulation using a von Frey filament $(0.4 \mathrm{~g})$ was measured. Data were calculated as the mean \pm S.E.M. values of 10 mice in each group. The statistical significance of the differences between the groups was assessed with one-way ANOVA followed by Dunnett's test. ${ }^{* \star \star} P<0.001$ when compared with the saline control group, ${ }^{\#} P<0.05$ when compared with the PTX-group.

1B). These observations are consistent with those of other reports showing paclitaxel to induce mechanical allodynia in mice $(4,19)$. The present study further demonstrated a significant attenuation of paclitaxel-induced mechanical allodynia by repeated milnacipran or fluvoxamine treatment.

Milnacipran and fluvoxamine are used for the treatment of acute and neuropathic pain as a supplementary analgesic $(16,21)$. There have been several reports indicating that milnacipran and fluvoxamine have significant antinociceptive effects against nociceptive and inflammatory pain $(20,22,27,28,30)$. Furthermore, milnacipran was more effective than SSRIs such as fluvoxamine, paroxetine, and citalopram in attenuating persistent pain (30) and neuropathic pain (9), and more effective than amitriptyline (TCAs) in attenuating cold allodynia $\left(4^{\circ} \mathrm{C}\right.$ cold plate stimuli) (1). However, the efficacy of milnacipran and fluvoxamine in attenuating paclitaxel-induced mechanical allodynia (tactile stimuli) is poorly understood. In this report, repeated but not acute administration of milnacipran significantly attenuated paclitaxel-induced mechanical allodynia. Repeated milnacipran administration was effective during both the development (days 0-4) and maintenance (days 5-9) of paclitaxel-induced mechanical allodynia. In contrast, repeated but not acute fluvoxamine administration resulted in a weak attenuation of paclitaxelinduced mechanical allodynia.

Milnacipran shows equivalent inhibitory activity at both NA and 5-HT neuronal reuptake systems (18). Obata et al. reported the involvement of both NA and 5-HT in the antiallodynic effect of milnacipran in a rat neuropathic pain model (20). However, the antiallodynic and antihyperalgesic effects of milnacipran in neuropathic pain models were predominantly attenuated by the noradrenergic system rather than by the serotonergic system at the spinal level $(9,28)$. Moreover, modulation of the noradrenergic system is likely to be more effective than that of the serotonergic system for the control of pain $(9$, $22,28,30)$. We confirmed that when administered concurrently with paclitaxel or after cessation of paclitaxel treatment, repeated milnacipran significantly attenuated paclitaxel-induced mechanical allodynia. On the other hand, repeated administration of fluvoxamine resulted in weak attenuation of paclitaxel-induced mechanical allodynia. Therefore, the antiallodynic effect of milnacipran in the paclitaxelinduced neuropathic pain model might be mediated primarily by the noradrenergic system rather than by the serotonergic system. 
Central glial cells such as astrocytes and microglia play a prominent role in pain modulation (17, 23). Spinal astrocytes and microglia have been found to play critical roles in facilitating central plasticity following both nerve injury and inflammation $(17,23)$. Indeed, spinal glial cells contribute to the development of paclitaxel-induced painful neuropathy in a rat model $(2,3)$. Recently, Zhang et al. reported that spinal astrocytes contribute to the pathogenesis of paclitaxel-induced painful neuropathy (31). The neuronal NA transporter is functionally expressed in cultured rat astrocytes and milnacipran potently inhibits NA uptake in these cells (10). The NA uptake system in astrocytes is sensitive to milnacipran, and the therapeutic effects of SNRIs may thus be mediated to some extent by their action on astrocytes. Together with these findings, our data suggest that chronic but not single milnacipran treatment attenuates paclitaxel-induced neuropathic pain that is, at least in part, through the inhibition of astrocyte activation.

In conclusion, chronic i.p. administration of paclitaxel at a once-daily dose of 1 or $2 \mathrm{mg} / \mathrm{kg}$ for 5 days induced mechanical allodynia which peaked on day 10 , decreased gradually, and largely subsided by day 20. This model may prove useful for further study of paclitaxel-induced neuropathic pain. We demonstrate a significant attenuation of paclitaxel-induced neuropathic pain by repeated milnacipran treatment. Importantly, this effect was observed not only during the development (days 0-4) but also during maintenance (days 5-9) of paclitaxel-induced neuropathic pain. The present results indicate that milnacipran may be a promising supportive therapeutic approach for paclitaxel-induced neuropathic pain.

\section{REFERENCES}

1. Berrocoso E, Mico JA, Vitton O, Ladure P, Newman-Tancredi A, Depoortère R and Bardin L (2011) Evaluation of milnacipran, in comparison with amitriptyline, on cold and mechanical allodynia in a rat model of neuropathic pain. Eur $J$ Pharmacol 655, 46-51.

2. Burgos E, Gómez-Nicola D, Pascual D, Martín MI, NietoSampedro M and Goicoechea C (2012) Cannabinoid agonist WIN 55,212-2 prevents the development of paclitaxelinduced peripheral neuropathy in rats. Possible involvement of spinal glial cells. Eur J Pharmacol 682, 62-72.

3. Cata JP, Weng HR and Dougherty PM (2008) The effects of thalidomide and minocycline on taxol-induced hyperalgesia in rats. Brain Res 1229, 100-110.

4. Costa R, Motta EM, Dutra RC, Manjavachi MN, Bento AF, Malinsky FR, Pesquero JB and Calixto JB (2011) Anti-nociceptive effect of kinin $\mathrm{B}_{1}$ and $\mathrm{B}_{2}$ receptor antagonists on peripheral neuropathy induced by paclitaxel in mice. $\mathrm{Br} J$
Pharmacol 164, 681-693.

5. Dharmshaktu P, Tayal V and Kalra BS (2012) Efficacy of antidepressants as analgesics: a review. J Clin Pharmacol 52, 6-17.

6. Dougherty PM, Cata JP, Cordella JV, Burton A and Weng HR (2004) Taxol-induced sensory disturbance is characterized by preferential impairment of myelinated fiber function in cancer patients. Pain 109, 132-142.

7. Flatters SJ and Bennett GJ (2004) Ethosuximide reverses paclitaxel- and vincristine-induced painful peripheral neuropathy. Pain 109, 150-161.

8. Flatters SJ and Bennett GJ (2006) Studies of peripheral sensory nerves in paclitaxel-induced painful peripheral neuropathy: evidence for mitochondrial dysfunction. Pain 122, 245257.

9. Ikeda T, Ishida Y, Naono R, Takeda R, Abe H, Nakamura T and Nishimori T (2009) Effects of intrathecal administration of newer antidepressants on mechanical allodynia in rat models of neuropathic pain. Neurosci Res 63, 42-46.

10. Inazu M, Takeda H and Matsumiya $T$ (2003) Expression and functional characterization of the extraneuronal monoamine transporter in normal human astrocytes. J Neurochem 84, 43-52.

11. Kawashiri T, Egashira N, Itoh Y, Shimazoe T, Ikegami Y, Yano T, Yoshimura M and Oishi R (2009) Neurotropin reverses paclitaxel-induced neuropathy without affecting antitumour efficacy. Eur J Cancer 45, 154-163.

12. Kesim M, Duman EN, Kadioglu M, Ulku C, Muci E, Kalyoncu NI and Yaris E (2006) Antinociceptive effects of fluoxetine and paroxetine with their related actions on glycemia in mice. Neuro Endocrinol Lett 27, 281-287.

13. Kesim M, Duman EN, Kadioglu M, Yaris E, Kalyoncu NI and Erciyes N (2005) The different roles of 5-HT(2) and 5 -HT(3) receptors on antinociceptive effect of paroxetine in chemical stimuli in mice. $J$ Pharmacol Sci 97, 61-66.

14. Matsumoto M, Inoue M, Hald A, Xie W and Ueda H (2006) Inhibition of paclitaxel-induced A-fiber hypersensitization by gabapentin. J Pharmacol Exp Ther 318, 735-740.

15. Merskey H and Bogduk N (1994) Classification of Chronic Pain: Descriptions of Chronic Pain Syndromes and Definitions of Pain Terms, 2nd edn. IASP Press, Seattle.

16. Micó JA, Ardid D, Berrocoso E and Eschalier A (2006) Antidepressants and pain. Trends Pharmacol Sci 27, 348-354.

17. Milligan ED and Watkins LR (2009) Pathological and protective roles of glia in chronic pain. Nat Rev Neurosci 10, 23-36.

18. Mochizucki D (2004) Serotonin and noradrenaline reuptake inhibitors in animal models of pain. Hum Psychopharmacol 19, S15-19.

19. Nieto FR, Entrena JM, Cendan CM, Pozo ED, Vela JM and Baeyens JM (2008) Tetrodotoxin inhibits the development and expression of neuropathic pain induced by paclitaxel in mice. Pain 137, 520-531.

20. Obata H, Saito S, Koizuka S, Nishikawa K and Goto F (2005) The monoamine-mediated antiallodynic effects of intrathecally administered milnacipran, a serotonin noradrenaline reuptake inhibitor, in a rat model of neuropathic pain. Anesth Analg 100, 1406-1410.

21. Onal A, Parlar A and Ulker S (2007) Milnacipran attenuates hyperalgesia and potentiates antihyperalgesic effect of tramadol in rats with mononeuropathic pain. Pharmacol Biochem Behav 88, 171-178.

22. Otsuka N, Kiuchi Y, Yokogawa F, Masuda Y, Oguchi K and Hosoyamada A (2001) Antinociceptive efficacy of antidepres- 
sants: assessment of five antidepressants and four monoamine receptors in rats. $J$ Anesth $\mathbf{1 5}, 154-158$.

23. Ren K and Dubner R (2010) Interactions between the immune and nervous systems in pain. Nat Med 16, 1267-1276.

24. Rogawski MA and Löscher W (2004) The neurobiology of antiepileptic drugs for the treatment of nonepileptic conditions. Nat Med 10, 685-692.

25. Sawynok J, Esser MJ and Reid AR (1999) Peripheral antinociceptive actions of desipramine and fluoxetine in an inflammatory and neuropathic pain test in the rat. Pain 82, 149158.

26. Schiff PB and Horwitz SB (1980) Taxol stabilizes microtubules in mouse fibroblast cells. Proc Natl Acad Sci USA 77, 1561-1565.

27. Schreiber S, Backer MM, Yanai J and Pick CG (1996) The antinociceptive effect of fluvoxamine. Eur Neuropsychopharmacol 6, 281-284.
28. Suzuki T, Ueta K, Tamagaki S and Mashimo T (2008) Antiallodynic and antihyperalgesic effect of milnacipran in mice with spinal nerve ligation. Anesth Analg 106, 1309-1315.

29. Woolf CJ and Mannion RJ (1999) Neuropathic pain: aetiology, symptoms, mechanisms, and management. Lancet 353, 1959-1964.

30. Yokogawa F, Kiuchi Y, Ishikawa Y, Otsuka N, Masuda Y, Oguchi K and Hosoyamada A (2002) An investigation of monoamine receptors involved in antinociceptive effects of antidepressants. Anesth Analg 95, 163-168.

31. Zhang H, Yoon SY, Zhang H and Dougherty PM (2012) Evidence that spinal astrocytes but not microglia contribute to the pathogenesis of paclitaxel-induced painful neuropathy. $J$ Pain 13, 293-303.

32. Zimmermann M (1983) Ethical guidelines for investigation of experimental pain in conscious animals. Pain 16, 109110. 